\title{
A CRITICAL COMPILATION OF ATOMIC OSCILLATOR STRENGTHS FOR CARBON, NITROGEN AND OXYGEN IONS
}

\author{
N. ALLARD*. (1), M.-C. ARTRU*, (1.2), T. LANZ* and M. IE DOURNEUF*, (3) \\ *Département Atomes et Molécules en Astrophysique, observatoiso de \\ Paris, Section de Meudon, F-92195 Meudon, France \\ "Institut d'Astronomie de I'Université de Lausanre. Cli-1290 \\ Chavannes-des-Bois, Switzerland
}

\begin{abstract}
Résumé
Nous avons entrepris une compilation de forces d'oscillateur pour les raies observées des ions de carbone, azote et oxygène. Cette compilation est achevée pour les spectres de la séquence isoélectronique du béryllium, C III, N IV et $\mathrm{OV}$. Les données théoriques de soixante publications (1966-1989) sont données en détail, ainsi que les résultats exhaustifs du projet OPACITY. On donne également une compilation des mesures de durée de vie des niveaux et leur comparaison aux résultats théoriques. La précision des différentes sources de données est analysée. Des moyennes pondérées des meilleures forces d'oscillateur théoriques sont proposées comme valeurs à adopter.
\end{abstract}

\begin{abstract}
We have undertaken a compilation of the oscillator strengths of observed lines for carbon, nitrogen and oxygen ions. It has been achieved for the Be-like spectra, C III, N IV and $\mathrm{O} V$. The theoretical data of sixty publications (1966-1989) are reported in detail, as well as the exhaustive results of the OPACITY project. The level lifetimes measured for these ions are also compiled and compared to the theoretical lifetimes. The accuracy is analysed according to the different data sources. Adopted gf-values are proposed from a weighted mean of the best theoretical results.
\end{abstract}

\section{1 - INTRODUCTION}

Carbon, nitrogen and oxygen are important elements in numerous scientific fields. Due to their large cosmic abundances $\left(10^{-4.3}, 10^{-4.0}\right.$ and $10^{-3.1}$ particles of $\mathrm{C}, \mathrm{N}$ and $\mathrm{O}$, relatively to hydrogen), there are specific astrophysical needs of reliable spectroscopic data concerning these atoms and their ions. In particular they contribute strongly to the opacity of stellar atmospheres and a correct determination of their radiative absorption is very important to construct coherent stellar atmosphere models (e.g. Seaton $/ 1 /$ ), these models being the basis of our understanding of the stellar evolution. Products of the CNO burning cycle of hydrogen are present on the stellar surface at some evolutionary stages and give important clues on this evolution. The diffusion of these elements in stellar envelopes and atmospheres must also be investigated with a correct estimation of radiative forces (e.g. Michaud /2/). Besides stars, low-density interstellar nebulae exhibit numerous emission lines due to forbidden transitions of these ions.

The critical compilation by Wiese et al /3/ provides oscillator strengths for these elements, but a lot of new data have been published later on. In particular many reliable theoretical results have been obtained, thanks to the development of sophisticated atomic structure calculations. Meanwhile numerous radiative lifetimes have been measured by the beam-foil technique, allowing an experimental determination of oscillator strengths for multiply-charged ions. In order to make this amount of new data easily available, especially for astrophysical applications, it appeared necessary to undertake an actualized compilation of oscillator strengths for all the atoms and ions of these important elements $\mathrm{C}, \mathrm{N}$ and $\mathrm{O}$. It gives the opportunity to include the recent theoretical results obtained in the context of the OPACITY project (Seaton

(1) URA 812 du CNRS

(2) Present address:

Ecole Normale Supérieure de Lyon, 46 Allée d'Italie, F-69364 Lyon Cedex 07, France

(3) UPR $261 \mathrm{du}$ CNRS 
$/ 1 /)$. The comparison between all previous results, either experimental or theoretical, and these new exhaustive data provides a test of their accuracy.

We present here our compilation of evaluated oscillator strengths for the observed lines of the Be-like isoelectronic spectra, C III, N IV, and O V (Allard, Artru, Lanz and Le Dourneuf, in press). This sequence has often been used to test the efficiency of new theoretical methods, because the four-electron atomic structures show critical correlation effects.

\section{2 - DESCRIPTION OF THE COMPILATION}

The purpose of this compilation is to provide an easy access to the existing data and to extract the best possible gf-values. This is achieved in two steps: every individual determination is reported in detail, with a qualitative indication on their expected accuracy, then adopted values are suggested and have been listed in a practical form for applications. The adopted values are justified from the critical discussion of the different theoretical methods. By means of the detailed compilation, any other choice about the final average could be adopted.

This compilation of oscillator strengths concerns all the observed lines of C III, N IV, and O V. All considered lines are permitted electric-dipole (E1) transitions, except a number of spin-forbidden intercombinations, due to departures from the pure LS coupling. Since most of the calculations are done assuming pure LS coupling, we have first compiled the total multiplet gf-values and intercombination lines are treated separately.

Although this compilation is restricted to the observed lines, it refers to a number of theoretical calculations which also consider additional weaker transitions, not observed but predicted either from observed levels, or from theoretical levels.

We started from the previous compilation on light elements by Wiese et al /3/. It contains oscillator strengths for 28,21 and 24 multiplets of $C$ III, N IV, and O V respectively: all of them come from two sets of theoretical calculations and are given with uncertainties of $25 \%$ to $50 \%$. The present compilation is based on more than one hundred new publications from 1966 to 1989. About half of them are theoretical and provide new data for 204, 190 and 140 multiplets of C III, N IV, and O V. The other ones report measurements of level lifetimes by the beam-foil technique. We have separately compiled the experimental lifetimes in order to compare them to those derived from the best set of theoretical oscillator strengths.

\section{3 - ANALYSIS OF THE THEORETICAL OSCILLATOR STRENGTHS}

With four electrons, the Be-like ions already show strong departures from the single-electron model, and still provide simple cases to test new techniques accounting for correlation effects. A total of 59 theoretical papers, published between 1966 and 1989, are listed for these spectra. They are briefly described in the compilation (Allard, Artru, Lanz and Le Dourneuf, in press) with emphasis on the current progresses of computational methods applied to C III, N IV and O V.

About one quarter of these theoretical works have been considered as superseded. Besides them, the most extensive studies are based on two standard codes of configuration interaction (CI): SUPERSTRUCTURE (Eissner and Nussbaumer /4/ which uses a basis of numerical orbitals solution of a scaled Thomas-Fermi (TF) potential usually varied in order to minimize the sum of the energies of the various atomic states of interest, and CIV3 (Hibbert /5/) which uses a set of Slater analytical orbitals that is varied in order to optimize the energy of individual states.

While the previous atomic structure methods are best to treat transitions involving valence electrons, the close-coupling method, for low-energy scattering calculation, is best to describe highly excited states, and this explains the broad scope of the OPACITY calculation (see Seaton /1/). The OPACITY results concerning the Be-like sequence have been computed by Tully, Berrington and Seaton (in preparation) and are presented by Tully (in these proceedings).

Since most calculations are based on similar physical approximations, their relative accuracy is mainly evaluated by comparison of the extensions and of the optimization of the various developments, as well as their convergence studies. Although the agreement between the length $(\mathrm{L})$ and velocity $(\mathrm{V})$ forms of the oscillator strengths is by no means a guaranty of the accuracy of an approximate treatment ( $L, V$ are identical in model potential calculations, for example), we consider it as an indication of the respective convergence of similar superposition-of-configuration studies. Since the velocity form is more sensitive to the correlations at short distances and more slowly convergent than the length form in most CI studies, especially in CC calculations, we have tabulated the $L$ results.

From this analysis, we have finally ordered the theoretical papers in three categories: (1) most accurate methods; (2) other CI calculations; and (3) superseded results. To estimate the best gf-value to be adopted for each multiplet, we chose an intermediate way between two opposite methods which consist either of extracting a number from the source which appears the most reliable one, or averaging all independent data. A weighted mean between a selected set of results was done, on the basis of the previous hierarchy of the theoretical methods. Those of the third category (col. 8) have been ignored. 
Table 1 presents a sample of the compilation: it concerns the low-excitation transitions of $\mathrm{O} V$. The gfvalues of observed transitions are given in different columns (col. 6, 7 and 8), according to the three categories of uncertainty which has been previously estimated from the degree of sophistication of the corresponding theoretical calculation. A special column (col. 5) is devoted to the results of the OPACITY calculation. Adopted values from the present compilation are proposed (col. 4) and those of the previous compilation of Wiese et al $/ 3 /$ are also recalled (col. 9 ).

In order to evaluate the overall accuracy of the adopted values, we have displayed in Fig. 1 the relative differences between the various theoretical gf-values of the first category and the adopted means for $O \mathrm{~V}$. These graphs are limited to the results of the first category (col.5 and 6) and the independent determinations are plotted with different symbols in order to show the absence of systematic errors. The general consistency appears to be better than $10 \%$, with exceptions for very weak lines (typically gf smaller than 0.001). Since a definite uncertainty cannot be established on the basis of theoretical results only, it is important to note that this estimated accuracy of $10 \%$ have been confirmed by the compilation of experimental measurements, as discussed below.

Total gf-value have been compiled for each multiplet and possible departures from the pure LS coupling were ignored. From some calculations which have been performed in intermediate coupling or in a fully relativistic approach, it appears that the gf-ratios inside multiplets are close to pure LS ones (within a few percents).

Because the weak singlet-triplet transitions are of special astrophysical interest, a separate compilation has been given for oscillators strengths of intercombination lines, which remain of the electric dipole (E1) type, but are due to term mixing due to spin-orbit. Since these transitions depend on weak correlation effects, the accuracy usually reached is smaller than for the allowed transitions.

Finally, we mention that a few studies have specifically focussed on forbidden (E2, M1, M2) transition probabilities. We have not compiled them, but just kept the corresponding papers in the reference list, with a precise description of their content (see Allard, artru, Lanz and Le Dourneuf, in press).

Table 1

Sample of the compilation of theoretical multiplet gf-values : the low-excitation transitions of $\mathrm{OV}$ (the abbreviated references are defined in the complete list given by Allard, Artru, Lanz and Le Dourneuf, in press).

\begin{tabular}{|c|c|c|c|c|c|c|c|c|c|c|c|c|c|}
\hline \multirow[t]{2}{*}{$E_{\mathrm{exc}}[\mathrm{eV}]$} & \multirow[t]{2}{*}{ Transition } & \multirow[t]{2}{*}{$\lambda_{\mathrm{m}}^{(\mathrm{\sigma})}[\mathrm{nm}]$} & \multicolumn{11}{|c|}{$g f_{\text {mult }}$} \\
\hline & & & \multirow{2}{*}{$\begin{array}{l}\text { Adopted } \\
0.514\end{array}$} & OPACITY & \multicolumn{8}{|c|}{ Other theoretical values } & Wie 66 \\
\hline 0.00 & $2 s^{2} S-2 p+p^{0}$ & 62.9730 & & $0.522 *$ & $\begin{array}{l}0.522 \\
0.509 \\
0.512 \\
0.525 \\
0.503 \\
0.515\end{array}$ & $\begin{array}{l}\text { Hib } \\
\text { Kib } \\
\text { Mos } \\
\text { Mul } \\
\text { Nus } \\
\text { Sim }\end{array}$ & $\begin{array}{l}74 \\
80 \\
76 \\
76 \\
79 \\
73\end{array}$ & $\begin{array}{l}0.52 \\
0.51 \\
0.51 \\
0.51 \\
0.51 \\
0.48\end{array}$ & $\begin{array}{ll}\text { Bur } & 72 \\
\text { Gla } & 79 \\
\text { Hum } 74 \\
\text { Lau } 78 \\
\text { Nic } 73 \\
\text { Nus } 72\end{array}$ & $\begin{array}{l}0.527 \\
0.524 \\
0.529 \\
0.64 \\
0.550 \\
0.742 \\
0.593 \\
0.53 \\
0.74 \\
0.536 \\
0.49 \\
0.499 \\
0.59 \\
0.560 \\
0.530 \\
0.45 \\
0.495 \\
0.517 \\
0.530\end{array}$ & $\begin{array}{l}\text { Arm } \\
\text { Che } \\
\text { Che } \\
\text { Czy } \\
\text { Faw } \\
\text { Fro } \\
\text { Gan } \\
\text { Gar } \\
\text { Gru } \\
\text { Joh } \\
\text { Lap } \\
\text { Lin } \\
\text { Mal } \\
\text { Mar } 8 \\
\text { Ser } \\
\text { Stee } \\
\text { Stew } \\
\text { Vic } \\
\text { Wat }\end{array}$ & $\begin{array}{l}7 \overline{6} \\
77 \\
79 \\
79 \\
78 \\
67 \\
79 \\
67 \\
66 \\
82 \\
66 \\
77 \\
75 \\
81 \\
86 \\
66 \\
75 \\
77 \\
75\end{array}$ & 0.53 \\
\hline 0.00 & $2 s^{21} s-3 p p^{0}$ & 17.21689 & 0.391 & 0.394 & & & & $\begin{array}{l}0.385 \\
0.37 \\
0.40 \\
0.37\end{array}$ & $\begin{array}{ll}\text { Gla } & 79 \\
\text { Hum } & 74 \\
\text { Lau } & 78 \\
\text { Nus } & 72\end{array}$ & $\begin{array}{l}0.45 \\
0.371 \\
0.374 \\
0.394 \\
0.381 \\
0.395 \\
0.400\end{array}$ & $\begin{array}{l}\text { Faw } 8 \\
\text { Fro } \\
\text { Gan } \\
\text { Lin } \\
\text { Mar } 8 \\
\text { Ser } 8 \\
\text { Stew }\end{array}$ & $\begin{array}{l}81 a \\
67 \\
79 \\
77 \\
81 \\
86 \\
75\end{array}$ & 0.59 \\
\hline 0.00 & $2 \mathrm{~s}^{2} \mathrm{~S}-3 \mathrm{~d}^{\prime} \mathrm{p}^{0}$ & 13.90289 & 0.046 & 0.058 & 0.031 & Hib & 80 & $\begin{array}{l}0.05 \\
0.05\end{array}$ & $\begin{array}{ll}\text { Gla } & 81 \mathrm{c} \\
\text { Lau } & 78\end{array}$ & & & & \\
\hline 0.00 & $2 \mathrm{~s}^{2} \mathrm{~S}-4 \mathrm{p}{ }^{1} \mathrm{P}^{0}$ & 13.55232 & 0.080 & 0.078 & & & & $\overline{0 . \overline{08}}$ & Lau 78 & $\begin{array}{l}0.114 \\
0.121 \\
0.116 \\
0.078 \\
0.121\end{array}$ & $\begin{array}{l}\text { Gan } 7 \\
\text { Lin } 7 \\
\text { Mar } 8 \\
\text { Ser } 8 \\
\text { Stew } 7\end{array}$ & & \\
\hline
\end{tabular}




\section{4 - EXPERIMENTAL LIFETIMES}

The beam-foil technique has provided measurements of radiative lifetimes for nearly fonty levels of cach ion, C III, N IV, and O V, the results being published in 43 different references since 1966. The compilation reports all of the measured lifetimes and the uncertainties given by the authors.

As already pointed out (e.g. Martinson /6/) there was a significant improvement in the reliability of measured lifetimes when the importance of cascading effects has been realized. In the most recent measurements, the ANDC method was applied to take into account the cascades (e.g. Reistadt $/ 7 /$ ) and they can claim accuracy ranges of $1 \%$ to $3 \%$. We have compared experimental and theoretical lifetimes, using the best results on both sides. An example is given in Tables 2 for $\mathrm{O} V$, where the last column gives the theoretical lifetimes. These have been calculated from the set of previously adopted gf-values and by using the OPACITY data to take into account decays through predicted transitions which may contribute to the lifetimes, even with weak branching ratios. It appears that differences between individual measurements are often outside the claimed uncertainty ranges, in particular for the earliest data, but the calculated lifetimes are in close agreement with the most reliable measurements, especially the recent ones which have been well corrected for cascading effects. This also appears in Fig.2 where all the lifetimes of O V, measured earlier or later than 1975 , are plotted versus the theoretical ones. The overall agreement of the best measurements remains within $10 \%$, while their uncertainties generally range from 3 to $10 \%$.

Therefore the performances independently achieved by theoretical and experimental methods lead to a comparable level of accuracy, while the previous comparison have shown their mutual agreement. For these reasons it appeared useless to use the experimental lifetimes to revise the previous theoretical adopted values .

Table 2

Sample of the compilation of lifetimes for the lower O V levels.

\begin{tabular}{|c|c|c|c|c|c|}
\hline level & $\begin{array}{c}\text { energy } \\
\text { (eV) }\end{array}$ & \multicolumn{2}{|c|}{$\begin{array}{l}\text { measured lifetime } \\
\text { (ns) }\end{array}$} & reference & calculated \\
\hline $2 \mathrm{p}{ }^{3} \mathrm{Po}_{0}$ & 10.16 & $\begin{array}{l}4.3 \\
5.26\end{array}$ & \pm 0.07 & $\begin{array}{l}\text { Dru } 71 \\
\text { Eng } 79\end{array}$ & \\
\hline $2 \mathrm{p} 3 \mathrm{po}_{1}$ & 10.18 & $\begin{array}{l}4.8 \\
4.3 \\
4.74\end{array}$ & $\begin{array}{l} \pm 0.5 \\
\pm 0.4 \\
\pm 0.07\end{array}$ & $\begin{array}{l}\text { Lew } 69 \\
\text { Dru } 71 \\
\text { Eng } 79\end{array}$ & \\
\hline $2 \mathrm{p}{ }^{3} \mathrm{PO}_{2}$ & 10.21 & $\begin{array}{l}5.4 \\
4.8 \\
5.36\end{array}$ & $\begin{array}{l} \pm 0.2 \\
\pm 0.5 \\
\pm 0.07\end{array}$ & $\begin{array}{l}\text { Lew } 69 \\
\text { Dru } 71 \\
\text { Eng } 79\end{array}$ & \\
\hline $2 p^{1} P^{\circ}$ & 19.69 & $\begin{array}{l}0.43 \\
0.417 \\
0.38 \\
0.49 \\
0.36\end{array}$ & $\begin{array}{l} \pm 0.05 \\
\pm 0.017 \\
\pm 0.02 \\
\pm 0.05 \\
\pm 0.04\end{array}$ & $\begin{array}{l}\text { Mar 71 } \\
\text { Irw 73 } \\
\text { Pin 74 } \\
\text { Kny 75 } \\
\text { Buc 76 }\end{array}$ & 0.35 \\
\hline $2 p^{2} 3 p$ & 26.50 & $\begin{array}{l}0.46 \\
0.45 \\
0.50 \\
0.40\end{array}$ & $\begin{array}{l} \pm 0.05 \\
\pm 0.03 \\
\pm 0.05 \\
\pm 0.04\end{array}$ & $\begin{array}{l}\text { Mar 71 } \\
\text { Pin 74 } \\
\text { Kny 75 } \\
\text { Buc 76 }\end{array}$ & 0.45 \\
\hline $2 p^{2} 1 D$ & 28.73 & $\begin{array}{l}3.0 \\
3.2 \\
3.2 \\
3.2 \\
3.2 \\
2.92 \\
2.7 \\
2.65\end{array}$ & $\begin{array}{l} \pm 0.2 \\
\pm 0.14 \\
\pm 0.2 \\
\pm 0.25\end{array}$ & $\begin{array}{l}\text { Bic } 69 \\
\text { Dru } 69 \\
\text { Cey } 70 \\
\text { Dru } 71 \\
\text { Mar 71 } \\
\text { Lin 72 } \\
\text { Pin } 74 \\
\text { Buc } 76\end{array}$ & 3.0 \\
\hline $2 p^{2} 1 s$ & 35.70 & $\begin{array}{l}0.29 \\
0.28 \\
0.32 \\
0.285\end{array}$ & $\begin{array}{l} \pm 0.03 \\
\pm 0.03 \\
\pm 0.03 \\
\pm 0.03\end{array}$ & $\begin{array}{l}\text { Mar 71 } \\
\text { Pin 74 } \\
\text { Kny 75 } \\
\text { Buc 76 }\end{array}$ & 0.26 \\
\hline $3 \mathrm{~s} 3 \mathrm{~S}$ & 67.82 & $\begin{array}{l}0.09 \\
0.060 \\
0.054\end{array}$ & $\begin{array}{l} \pm 0.01 \\
\pm 0.006 \\
\pm 0.005\end{array}$ & $\begin{array}{l}\text { Buc } 72 \\
\text { Kny } 75 \\
\text { Buc } 76\end{array}$ & 0.055 \\
\hline
\end{tabular}




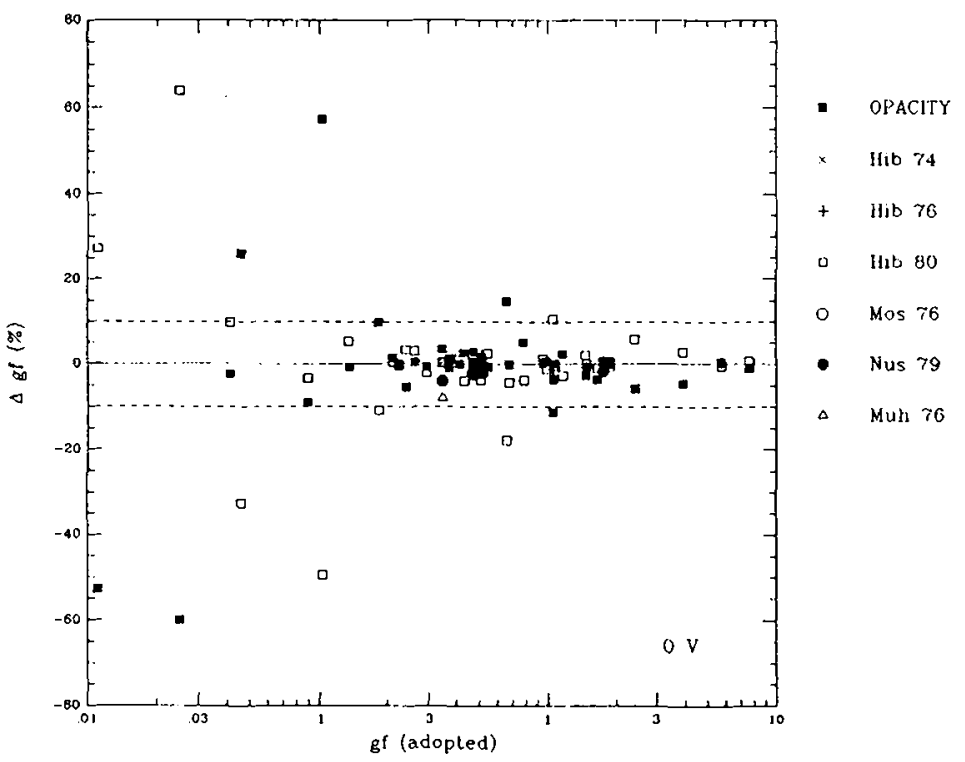

Fig. 1 - Relative difference between the most accurate published gf-values and the adopted ones for $\mathrm{OV}$ (a difference of $10 \%$ is indicated by the dashed lines).

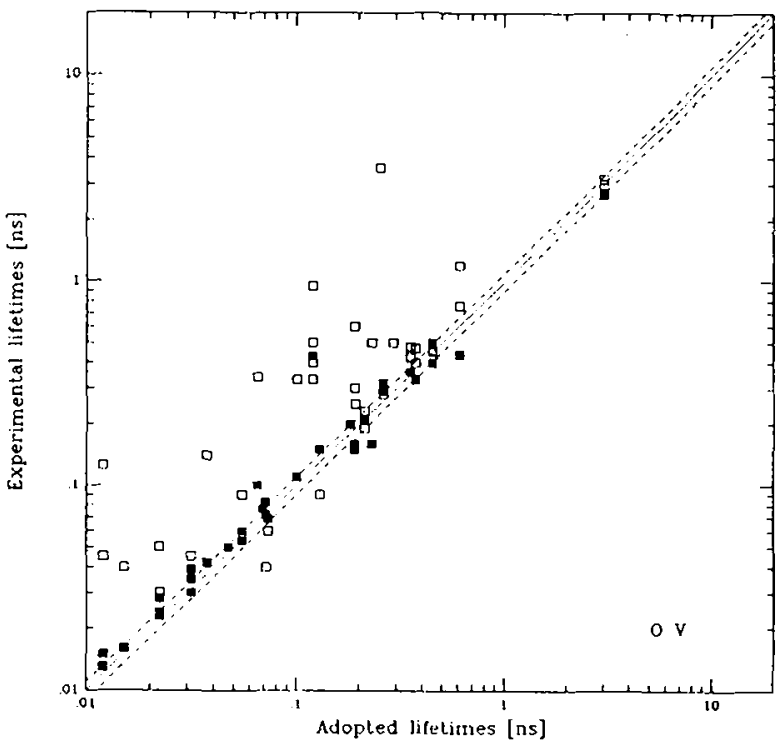

Fig. 2 - Comparison between the experimental lifetimes and those calculated from the adopted theoretical oscillator strengths for $\mathrm{O} V$ (filled squares represent the most recent results accounting for cascades; a difference of $10 \%$ is indicated by the dashed lines). 


\section{5 - TABLES OF ADOPTED LINE OSCILLATOR STRENGTHS}

Using the previous compiled data, we have prepared a set of complete line lists of the spectra $C$ III, $\mathrm{N} I V$, and $\mathrm{O}$ V, giving the new adopted gf-values (Allard, Artru, Lanz and Le Dourneuf, in press). The values of $\log$ gf are given for all individual observed lines. Most of them have been calculated from the adopted multiplet gf-values derived from the best theoretical works. A pure LS-coupling is assumed and the intercombination lines which have been directly compiled have been added.

\section{6 - CONCLUSION}

This compilation summarizes the recent progress concerning oscillator strengths of the three Be-like spectra C III, N IV and O V. The data are presented in a convenient form for applications. The total number of observed multiplets which are given with a reliable gf-value has increased by an order of magnitude from 1966 to 1989 . The accuracy of the gf-values has also been significantly improved since 1966 and could be estimated in the 10\% range for most of the transitions. This is attested by the internal consistency between the best theoretical results and by their agreement with the most recent lifetime measurements. The reliability of the OPACITY results has been emphazised, showing that extensive atomic calculations has become a powerful way to produce accurate oscillators strengths.

AKNOWLEDGments. We thank Professor M.J. Seaton for his supporting interest and his helpful comments about this work. We are also indebted to Drs K.A. Berrington and J. Tully for allowing us to quote their theoretical results in advance of publication, and to Dr A.K. Pradhan for sending us the corresponding files.

\section{REFERENCES}

/1/ Seaton, M. : 1987, J. Phys. B 20, 6363

R/ Michaud, G. : Physica Scripta1987,36, 112

B) Wiese, W.L., Smith, M.W., Glennon, B.M. : 1966, NSRDS-NBS4 Atomic Transition Probabilities, Hydrogen through Neon (US government, Printing Office, Washington, D.C.)

14/ Eissner, W., Nussbaumer, H.: 1969, J. Phys. B 2, 1028

15/ Hibbert, A.: 1975, Comput. Phys. Commun. 9, 141

16/ Martinson, I. : 1989, Rep. Prog. Phys. 52, 157

III Reistadt, N., Martinson, I. : 1986, Phys. Rev. A 4, 1986 